Table VI

Semi-Product Usage of Gold in Western Europe and the U.S.A. in 1980. Estimaled from $(2,4)$

\begin{tabular}{l|r|r|r|r}
\hline \multicolumn{1}{c|}{ Semi-product } & \multicolumn{2}{c|}{ W. Europe, } & \multicolumn{2}{c}{ U.S.A., } \\
& $t$ & $\%$ & $t$ & $\%$ \\
\hline $\begin{array}{l}\text { Electroplating salts } \\
\text { Metallurgical products }\end{array}$ & 28.2 & 66.5 & 39 & 59 \\
$\quad$ (wire, leaf, etc.) & 6.3 & 14.9 & 3 & 4.6 \\
Thick film pastes/ & & & & \\
liquid golds/ & & & & \\
$\quad$ chemicals & 1.9 & 4.5 & 6 & 9 \\
Brazing alloys & 2.0 & 4.7 & 5 & 7.7 \\
Rolled gold & 4.0 & 9.4 & 13 & 19.7 \\
Total & 42.4 & 100.0 & 66 & 100.0
\end{tabular}

distinct types of consumer demand. Within the costume jewellery segment growth prospects for consumption of gold appear to be favourable.

\section{Semi-Product Usage}

Although some reference has been made above to the various forms of semi-product in which gold is supplied and used, the subject is worthy of brief discussion and analysis here: From Table VI, it can be clearly seen that, excluding carat jewellery and dental alloys, approximately two-thirds of the gold consumed in industrial uses in the U.S.A. and Western Europe is in the form of electroplating salts, of which the most widely used is potassium gold(I) cyanide. Rolled gold occupies second place in the U.S.A., reflecting its traditional popularity in that country, although there are indications that consumption of this semi-product may decline in the coming years partly because of its inability to accommodate intricate designs which are beginning to find favour. In Western Europe, the lower consumption figure for rolled gold is clearly demonstrated.

\section{References}

1. 'Gold 1981' Consolidated Gold Fields Ltd , London, 1981

2. C. Michalopoulos, 'U.S. Gold Use in Industrial Applications', Michalopoulos and Parker, Washington, D.C., May, 1981, confidential report to International Gold Corporation Ltd.

3. C. Michalopoulos, 'U.S. Gold Use in Decorative Applications', Michalpoulos and Parker, Washington, D.C., June, 1981, confidential report to International Gold Corporation Ltd.

4. C.G. Wedgewood, 'The Market and Applications in Western Europe for Gold and it Terivatives in the Electrical and Electronic Industries', C.G. Wedgewood and Co., London, July, 1981, confidential report to International Gold Corporation Ltd.

5. 'Decorative and Other Industrial Uses of Gold in Western Europe', Battelle, Geneva, June, 1981, confidential report to International Gold Corporation Ltd

6. 'Spotlight on Connectors', supplement to Electronic News, 1981, November 9.

\title{
Anodic Oxide Growth on Gold
}

The bulk of the important work on the electrochemical oxidation of gold published up to 1980 has been reviewed by M.J. Nicol (Gold Bull., 1980,13, (2), 46-55 and (3), 105-111). In discussing the thermodynamic predictions and interpretations of the Pourbaix potential/pH diagram for gold, Nicol pointed out that $\mathrm{Au}_{2} \mathrm{O}_{3}$, the most stable trivalent gold oxide, should have a wide region of stability, whereas none exists for either $\mathrm{Au}(\mathrm{I})$ or $\mathrm{Au}(\mathrm{II})$ oxides.

Although the early work on the electrochemical oxidation of gold (before the last decade) was characterized by a lack of sensitivity and resolution inherent in the galvano-static methods in use at the time, a number of features relating to anodic oxide growth on gold were recognized and these have been, in part, confirmed by more recent research using cyclic voltammetry and other sophisticated techniques. The generally accepted conclusion was that, in noncomplexing acid solutions, with increasing electrode potential, chemisorption of oxygen occurs followed by monolayer coverage and finally growth of a bulk oxide layer of composition corresponding closely to that of $\mathrm{Au}_{2} \mathrm{O}_{3}$. In alkaline solutions the final oxide layer was thought to consist of $\mathrm{Au}(\mathrm{OH})_{3}$.

Later and more refined work has shown that the process is more complex than previously thought. A.J. Arvia et al. (J. Electroanal. Chem., 1975, 59, 239) considered that in non-complexing acids, initial formation of an adsorbed $\mathrm{AuOH}$ species is followed either by further oxidation to a higher 'oxide' or by chemical disproportionation to a more stable surface oxide, the stoichiometry of which approximates closely to $\mathrm{Au}_{2} \mathrm{O}_{3}$.

In a recent paper L.D. Burke and M. McRann (J. Electroanal. Chem., 1981, 125, 387-399) report on the electrochemical oxidation of gold in non-complexing alkaline solutions at potentials which are sufficiently high to cause oxide films to form on the gold surface. Thick films (of the order of 100 monolayer equivalents at $2.3 \mathrm{~V}$ ) formed under steady-state polarization conditions and, like those formed in acid solutions, were duplex in character. The layer consisted of a compact, largely anhydrous, inner film at the metal surface, and a much thicker, porous, highly hydrated outer film at the oxide/solution interface. The growth of oxide under alkaline conditions is slower than that in acid medium and reaches a limiting value. The outer film is reduced less easily and oxygen evolution is inhibited in the thick film region at about $2.35 \mathrm{~V}$. Changes in oxygen coordination of cations in the outer region of the compact layer under conditions of thick film growth are assumed by Burke and McRann to enhance the conversion of material in the outer region of this layer to the more highly hydrated form.

Interestingly, these authors discuss possible similarities between the thick oxide films which have been grown anodically on other transition metals of practical significance and the hydrous oxide layer formed on gold. It is intriguing to speculate on the possibility of the current research on anodic polarization of gold electrodes leading to the formation of stable oxide films with interesting electrochromic, charge storage, electrocatalytic, resistive and other properties which could be of industrial significance. 